\title{
Optimization of the Method of Constructing Ecological Security Pattern with Rapid Urban Expansion
}

\author{
Ke $\mathrm{Li}^{1}$, Tiantian $\mathrm{Yu}^{*}, J i \mathrm{Li}^{2}$, Chengling Cui ${ }^{1}$ and Song $\mathrm{Wu}^{2}$ \\ ${ }^{1}$ Beijing Geoway Space Information Co., Ltd., Beijing 100040, China; \\ ${ }^{2}$ Wuhan Geoway Space Information Technology Research Institute Co., Ltd., Wuhan 430076, China
}

\begin{abstract}
The contradiction between urban development and ecological environment protection is particularly obvious in rapidly expanding urban areas. It is necessary to build a reasonable urban ecological security pattern. to balance urban expansion and ecological security. This paper takes Zhengzhou as an example, based on spatial principal component analysis, a comprehensive assessment of urban ecological security is carried out by using natural characteristics, human characteristics, and natural and human interaction characteristics, and ecological sources are extracted; Based on the ecological corridors extracted by the minimal cumulative resistance (MCR) model, the edge-betweenness index is used to optimize the model, and redundant corridors are eliminated, the first-level ecological corridors and ecological nodes on the first-level ecological corridor are extracted. Through experiments, 25 important ecological sources, 24 first-level ecological corridors, and 32 ecological nodes were extracted. Important ecological sources, first-level ecological corridors and ecological nodes constitute the basic ecological security pattern of Zhengzhou, which effectively alleviate the contradiction between rapid urban expansion and ecological protection, and ensuring urban ecological security at the same time.
\end{abstract}

\section{Introduction}

With the rapid economic growth and expansion of cities, a series of ecological problems such as the reduction of habitat patches, serious landscape fragmentation, and loss of biodiversity have also arisen [1-2].How to scientifically manage urban spatial growth and balance the contradiction between rapid urban expansion and ecological protection is an important guarantee for sustainable urban development. In the 1990s, Professor Kongjian Yu of Peking University and his research team clearly proposed to deal with various problems caused by rapid urbanization in response to China's severe man-land relationship. The core solution was to establish a national ecological security pattern[3]

Minimal cumulative resistance (MCR) is a common model to select ecological corridors and the construction of ecological security patterns[4]. However the application to the construction of ecological corridors in larger areas has corridor redundancy problems [5].

\footnotetext{
Corresponding author: m13618650845_1@163.com
} 
Under the current situation of rapid urban expansion, land resources are in short supply, and the construction of ecological corridors should eliminate redundant corridors while ensuring the safety of the urban ecological pattern. In ecology, edge-betweenness is to calculate the frequency of a certain section of corridor used by biological flow and landscape flow, which can describe the frequency and importance of ecological corridor in the ecosystem. Therefore, based on the MCR model, this paper introduces the betweenness index and extracts irreplaceable ecological corridors as the first-level corridors [6] to construct ecological corridors .which can take into account urban expansion and ecological security, and provide a reference for the construction of a rapidly expanding urban ecological security pattern.

Zhengzhou City is a typical city with rapid expansion in central China. Its built-up area has increased from $133.2 \mathrm{~km} 2$ in 2000 to $443 \mathrm{~km} 2$ in 2016 , an expansion of 3.3 times [7]. In this process, the contradiction between urban construction and the ecological environment has gradually increased [8]. So this paper takes Zhengzhou City as an example to study and optimize the method of constructing the ecological security pattern of the rapidly expanding city.

\section{Research method}

The ecological security pattern consists of ecological sources, ecological corridors and ecological nodes. The ecological source area is the core plate that can maintain the stability of the ecosystem and has an ecological promotion effect in the region. Ecological corridors are important channels for interconnection and diffusion of urban ecosystems [9]. In the spatial model, the ecological corridor is the line of resistance valleys between adjacent ecological sources. The ecological node is the tangent point of the equal resistance line formed by two adjacent ecological sources as the center. It is a key node connecting the ecosystem and a relatively fragile point in the ecosystem ${ }^{[10]}$. It needs to be strengthened to repair and focus on protection.

\subsection{Ecological source identification based on spatial principal component analysis}

In this paper, evaluation factors are selected from three aspects of natural, man-made, natural and man-made synergistic factors, and spatial principal component analysis is used to analyze the spatial analysis of the evaluation factors, and the variance contribution rate corresponding to each principal component is used to express the weight of each evaluation factor. Use ArcGIS to perform a weighted summation of the principal components. The summation result is reclassified into 5 levels using the natural breakpoint method, and the patch with the highest ecological safety is taken as the ecological source ${ }^{[11]}$.

$$
I_{e s}=\sum_{n=1}^{j} P_{m n} w_{n}
$$

$I_{e s}$ is the ecological safety index, $P_{m n}$ is the $\mathrm{n}$-th index factor of the $\mathrm{m}$-th grid unit, $w_{n}$ is the weight of each factor, and $j$ is the index factors.

The selected evaluation factors are:

1) Natural factors: There are 3 evaluation factors extracted based on natural factors: elevation (DEM), slope and landscape connectivity. DEM and slope are the natural bases for the preservation of ecological sources; Landscape connectivity describes how easy it is for the landscape to flow with ecological elements, and is an important indicator to measure the ecological process. Good landscape connectivity is helpful to the effective realization of ecological functions. 
2) Man-made factors: Man-made factors include road density and luminous intensity. Road density reflects the intensity of urban infrastructure, and luminous intensity reflects the level of urbanization, urban economic conditions, population density and many other human activity factors, which can well represent the intensity and scope of human activities .

3) Natural and man-made synergistic evaluation factors: Natural and man-made synergistic factors include normalized vegetation index (NDVI), land use/cover, and habitat quality.

\subsection{Extracting ecological corridors based on the MCR model}

Minimal cumulative resistance describes the minimum cost path of cumulative resistance between ecological sources. It's formula ${ }^{[12]}$ is as follows:

$$
\operatorname{MCR}=f \min \sum_{j=n}^{i=m} \times R_{i}
$$

MCR is the minimum cumulative resistance value, $f$ is the positive correlation function between a certain point and the minimum cumulative resistance and the ecological process. $D_{i j}$ is the spatial distance from the ecological source $\mathrm{j}$ to the grid unit $\mathrm{i}, R_{i}$ is the correction resistance coefficient of the $i$ grid unit.

The construction of the resistance surface is based on the weighted result of the spatial principal component analysis as the resistance evaluation element, and the cost distance tool of ArcGIS is used to construct the spatial resistance surface.

The natural breakpoint method with the smallest difference within the group and the largest difference between groups divides the resistance surface into five levels: lowest resistance, lower resistance, medium resistance, higher resistance, and highest resistance. Ecological corridors are passages that pass through the lowest resistance level and connect to ecological sources.

\subsection{Extraction of first level ecological corridors based on the betweenness index}

The edge betweenness is defined as the proportion of the number of paths passing through a certain edge among all the shortest paths in the network to the total number of shortest paths, reflecting the role and influence of a certain edge in the entire network.

This paper abstracts the ecological sources and corridors into a network graph composed of nodes and edges, and introduces the edge betweenness index to extract the first-level ecological corridors [13].The formula is as follows:

$$
\operatorname{Betweenness}(l)=\sum_{s \neq t \in v} \frac{\delta_{s t}(l)}{\delta_{s t}}
$$

$l$ represents any edge in the network graph, s and t respectively represent two different nodes in the network graph, $\delta_{s t}(l)$ represents the number of shortest paths from node $\mathrm{s}$ to node $\mathrm{t}$ through edge 1 , and $\delta_{s t}$ is the number of shortest paths between $\mathrm{s}$ and t nodes. 


\section{Results and analysis}

\subsection{Extraction and optimization of ecological sources}

Landscape connectivity evaluation factors and habitat quality evaluation factors are calculated based on land use/cover results. The results of land use/cover are shown in Figure 1:

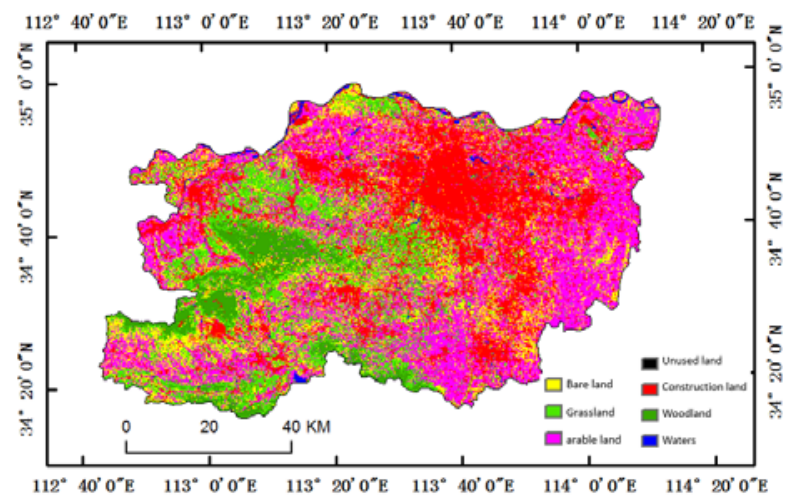

Fig. 1. Land use /cover map of Zhengzhou

The spatial pattern of a single evaluation factor reflects the impact of different ecological processes on ecological security. In this paper, the natural breakpoint method is used to divide the single evaluation factor into 1-9 grades. For land use/cover, based on experience, forest land is divided into first level, grassland, water area and grassland are divided into second level, cultivated land is divided into 7th level, bare land is divided into 8th level, and construction land is divided into 9th level.

The classification results are shown in the following table:

Table 1. Extraction and classification results of a single evaluation factor

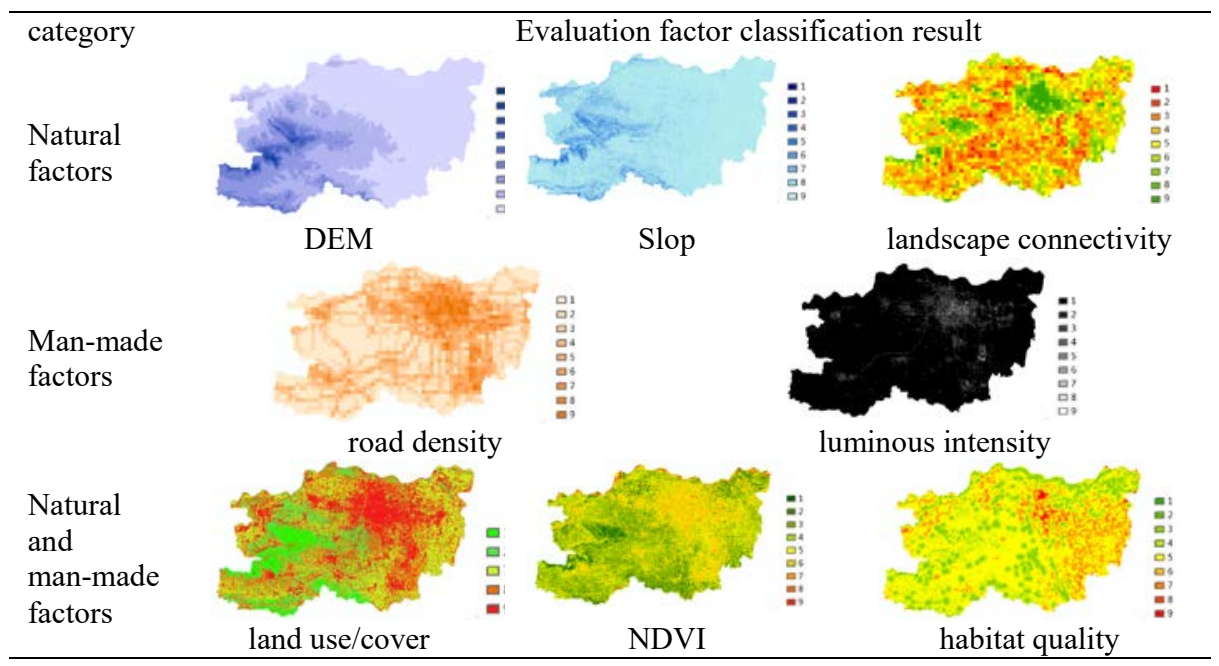

Based on the reclassification results of a single evaluation factor, using spatial principal component analysis, 8 principal components are generated. The first 5 principal components can well reflect the spatial distribution of ecological sources in the study area. 
The original evaluation factor loading corresponding to each principal component is shown in Table 2.

Table 2. Space principal component load matrix

\begin{tabular}{lllllllll}
\hline Evaluation factors & PC1 & PC2 & PC3 & PC4 & PC5 & PC6 & PC7 & PC8 \\
\hline DEM & 0.218 & 0.026 & 0.145 & 0.179 & 0.751 & -0.285 & -0.503 & -0.003 \\
Slop & 0.139 & 0.065 & 0.098 & 0.152 & 0.415 & -0.170 & 0.862 & 0.001 \\
connectivity & -0.021 & 0.475 & 0.842 & -0.097 & -0.099 & 0.182 & -0.028 & 0.108 \\
luminous intensity & 0.081 & -0.153 & -0.034 & -0.014 & -0.015 & -0.035 & 0.004 & 0.983 \\
road density & 0.422 & -0.743 & 0.440 & 0.120 & -0.206 & -0.012 & 0.014 & -0.137 \\
habitat quality & 0.121 & -0.090 & -0.101 & 0.062 & 0.331 & 0.924 & 0.011 & 0.011 \\
NDVI & 0.275 & -0.053 & -0.036 & -0.942 & 0.166 & -0.041 & 0.042 & -0.045 \\
land use/cover & 0.811 & 0.427 & -0.232 & 0.173 & -0.273 & -0.003 & -0.037 & -0.010 \\
\hline
\end{tabular}

It can be seen from Table 2 that the load of the connectivity evaluation factor is higher in the third principal component. Which indicates that the connectivity of the natural evaluation factor has a certain impact on the regional ecological source. The road density in the man-made evaluation factor ranks second in the load of the first principal component, and is the highest value, which indicating that the road density has a greater impact on the regional ecological source. Among the evaluation factors of natural and man-made synergistic factors, land use/cover is the high value of the first principal component, and the NDVI and habitat quality are the high values of the fourth and sixth principal components, which shows that the evaluation factor of natural and man-made synergy is an important factor affecting the regional ecological source.

Taking the contribution rate of each principal component as the weight, summing them and using the natural breakpoint method to reclassify, the ecological safety classification results of Zhengzhou City are obtained, as shown in Figure 2.

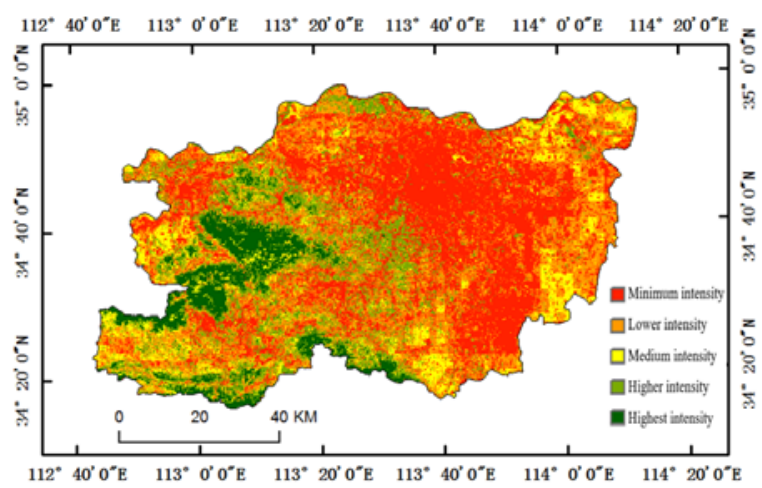

Fig. 2. Zhengzhou Ecological Safety Rating Map

Delete ecological security areas with an area of less than 500 square meters, and divide the highest ecological security areas as ecological sources, then we will get the spatial distribution map of the ecological source area in Zhengzhou.

According to the size of the ecological source area and the degree of aggregation and dispersion, this paper divides the ecological source area into a primary ecological source area and a secondary ecological source area (Figure 3). The primary ecological source area is larger and more concentrated, while the secondary ecological source area is small and 
distributed independently. It can be seen from Figure 3 that there are 13 primary ecological sources and 12 secondary ecological sources.

The primary ecological source is the main source for coordinating the urban ecosystem, and the secondary ecological source suffers from a large cumulative resistance, which is the areas that need to be expanded and optimized.

From the perspective of spatial distribution, the secondary ecological source located along the Yellow River is an important area for ecological supply in the eastern part of the city. Therefore, expanding the ecological source here is a shortcut to optimize the urban ecological security pattern.

\subsection{Ecological corridor extraction based on MCR model}

Based on the minimal cumulative resistance model, the cost distance module of ArcGIS is used to evaluate the spatial resistance of the ecological source traversing, and the spatial resistance surface are constructed. The ecological corridor is shown as a low-resistance channel between adjacent ecological sources on the spatial resistance map. The extracted ecological corridor is shown in Figure 3.

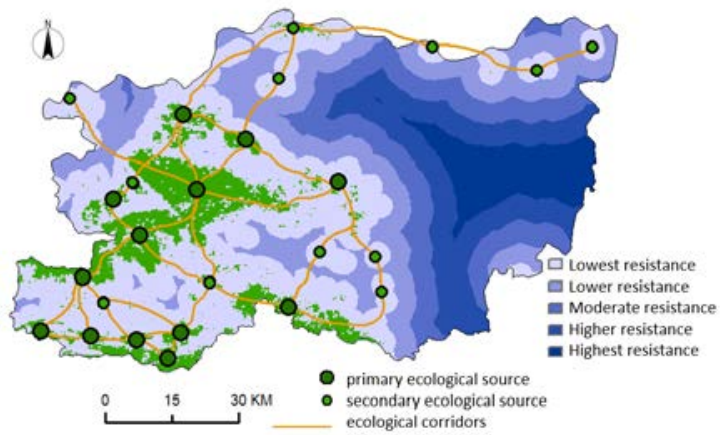

Fig. 3. Ecological corridor results extracted based on MCR model

There are a total of 38 ecological corridors extracted based on the MCR model. It can be seen from Figure 3 that the ecological corridors have redundancy in the spatial structure.

\subsection{Ecological corridor optimization based on edge-betweenness}

This paper abstracts the ecological source points and ecological corridors as the nodes and connecting edges of the network, and calculates the betweenness of the corridor. The larger the betweenness and the higher the usage frequency of the corresponding corridor, the more important it is in the network.Based on the edge-betweenness, this paper extracted 24 first-level ecological corridors, as shown in Figure 5(b).

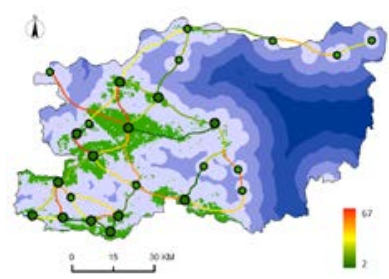

(a)edge-betweenness

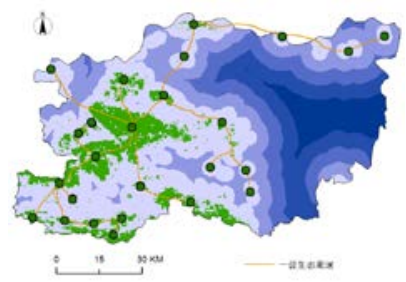

(b)first-level ecological corridors

Fig. 4. Ecological corridor results extracted based on edgebetweenness 
Compared with the ecological corridors extracted by the unoptimized model, the number of first-level corridors extracted after optimization is reduced. After optimization, the total length of the corridor was reduced from $577.95 \mathrm{~km}$ to $369.57 \mathrm{~km}$, a reduction of $36.06 \%$. After optimization, it can alleviate the contradiction between rapid urban expansion and ecological protection while ensuring the basic ecological security structure, maintaining the connectivity between ecological sources, and preventing the ecological environment from continuing to be fragmented.

\subsection{Establish ecological security pattern}

Ecological nodes are springboards for the connections and circulation between ecological sources, and they play a key role in the migration or spread of species. A total of 32 ecological nodes have been identified in this paper (Figure 5). It can be seen from Figure 5 that the cumulative resistance of some ecological nodes is relatively large, especially the ecological nodes located on the corridor connecting the secondary ecological source. For ecological nodes distributed on cultivated land types, appropriate conversion of farmland to forests can be carried out according to actual conditions; for ecological nodes distributed on construction land, it can be considered as an urban ecological park.

Improving and optimizing the ecological nodes can not only effectively reduce the ecological resistance on the ecological corridors, but also promote the flow of species through the ecological corridors. Which is an effective measure to improve the status quo of the urban ecosystem, enhance the exchange of information within the ecosystem, and promote the sustainable and healthy development of the urban ecosystem

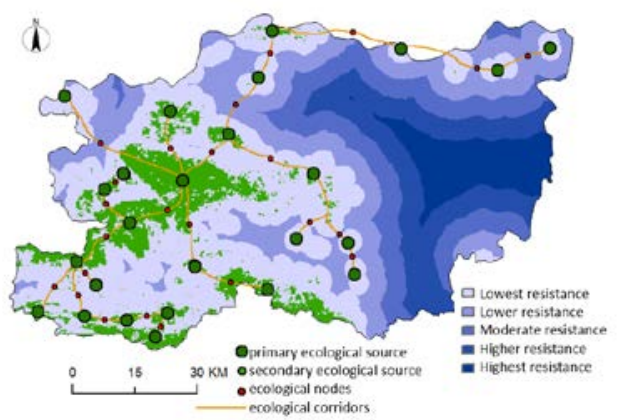

Fig. 5. Ecological security pattern

\section{Conclusion}

This paper comprehensively considers the three factors of nature, man-made, and natural and man-made synergy to extract the impact factors of urban ecological security, and identify the ecological source.Then based on the extraction of ecological corridors by the MCR model, the edge betweenness index was introduced to eliminate redundant corridors, and optimize the ecological security pattern of Zhengzhou City. The research showed that:

1) On the whole, Zhengzhou City has relatively low ecological security and the ecological sources are unevenly distributed. From the perspective of spatial distribution, the secondary ecological source located along the Yellow River is a potentially important area for urban ecological supply. Therefore, expanding the ecological source here is a shortcut to optimize the urban ecological security pattern. 
2) This paper abstracts the ecological security pattern as a network composed of ecological source points and ecological corridors. The introduction of the betweenness index can effectively eliminate redundancy and optimize the construction of ecological corridors. Using the usage frequency represents the importance of ecological corridors, to screen the first-level ecological corridors, and the ecological security pattern constructed thereby is more scientific.

3) An ecological security pattern constructed based on ecological sources, primary ecological corridors and ecological nodes, while ensuring the ecological security of the city and leaving more space for urban expansion, which can provide guidance for urban planning and construction..

\section{References}

[1] Lovell S T, Taylor J R, Supplying urban ecosystem services through multifunctional green infrastructure in the United States. Landscape Ecology, 2013,28(8):1447-1463.

[2] Fang Chuanglin, Zhou Chenghu, Gu Chaolin, Chen Liding, Li Shuangcheng. Theoretical Framework and Technical Path for Analyzing the Coupling Effect of Urbanization and Ecological Environment in Megacities [J] .Acta Geographica Sinica, 2016,71 (04): 531- 550.

[3] 2017-11-12. https://baike.so.com/doc/2092852-2213897.html

[4] Yang Liangjie, Wang Jing, Wei Wei, Yang Zecheng.Construction and optimization of the ecological security pattern of arid inland river basins: Taking Shiyang River Basin as an example[J/OL].Acta Ecologica Sinica,2020(17):1-13[2020-07-19.

[5] Alexandre Rosa dos Santos,Emanuel França Araújo,Quétila Souza Barros,Milton Marques Fernandes,Márcia Rodrigues de Moura Fernandes,Taís Rizzo Moreira,Kaíse Barbosa de Souza,Evandro Ferreira da Silva,Jeferson Pereira Martins Silva,Jeangelis Silva Santos,Dâmaris Billo,Rodrigo Freitas Silva,Glícia Silvania Pedroso Nascimento,Simony Marques da Silva Gandine,André Alves Pinheiro,Wilian Rodrigues Ribeiro,Morgana Scaramussa Gonçalves,Samuel Ferreira da Silva,Adriano Posse Senhorelo,Fabricio Dias Heitor,Leandro Christo Berude,Lucas Arthur de AlmeidaTelles. Fuzzy concept applied in determining potential forest fragments for deployment of a network of ecological corridors in the Brazilian Atlantic Forest[J]. Ecological Indicators,2020,115.

[6] Liu Fei. Vulnerability analysis and coping strategies of regional road network [D]. Chongqing Jiaotong University, 2019

[7] Xu Jing. Study on the Spread of Zhengzhou City's Boundary [J]. Chinese and Foreign Entrepreneurs, 2020 (02): 224-225.

[8] Zhang Baowei. Eco-environmental quality evaluation and system design based on RSEI model [D]. Zhengzhou University, 2018.

[9] Chaudhary Khushboo,Saraswat Pankaj Kumar,Khan Suphiya. Improvement in fluoride remediation technology using GIS based mapping of fluoride contaminated groundwater and microbe assisted phytoremediation.[J]. Ecotoxicology and environmental safety,2019,168.

[10] Zhou Yan, Chen Yao, Deng Shaopo, Wan Jinzhong, et al. Spatial principal component analysis and ecological risk assessment of heavy metals in farmland soil of a lead-zinc mining area in southwestern China[J]. Environmental Science, 2018, 39(06): 2884-2892. 
[11] Xu Kai, Yu Tiantian, Qin Kun, etc. The atmospheric haze effect of the "source" and "sink" landscape of multi-source remote sensing data taking into account the scale effect[J]. Environmental Science, 2017, 38(12):4905-4912.

[12] Huang Jiuming,Hu Yecui,Zheng Fangyu. Research on recognition and protection of ecological security patterns based on circuit theory: a case study of Jinan City.[J]. Environmental science and pollution research international,2020.

[13] Li Yanyan. Research on Urban Spatial Expansion Based on Ecological Security Pattern [D]. Northwest University, 2019 